\title{
Editorial
}

\section{Heuristic thinking: interdisciplinary perspectives on medical error}

\author{
Annegret F. Hannawa \\ Faculty of Communication Sciences, Institute of Communication and Health, University of Lugano, \\ Switzerland
}

Approximately 43 million adverse events occur across the globe each year at a cost of at least 23 million disability-adjusted life years and $\$ 132$ billion in excess health care spending, ranking this safety burden among the top 10 medical causes of disability in the world. ${ }^{1}$ These findings are likely to be an understatement of the actual severity of the problem, given that the numbers merely reflect seven types of adverse events and completely neglect ambulatory care, and of course they only cover reported incidents. Furthermore, they do not include statistics on children and incidents from India and China, which host more than a third of the world's population. Best estimates imply that about two thirds of these incidents are preventable. Thus, from a public health perspective, medical errors are a serious global health burden, in fact ahead of high-profile health problems like AIDS and cancer.

Interventions to date have not reduced medical errors to satisfactory rates. Even today, far too often, hand hygiene is not practiced properly (even in developed countries), surgical procedures take place in underequipped operating theaters, and checklists are missing or remain uncompleted. The healthcare system seems to be failing in managing its errors it is costing too much, and the complexity of care causes severe safety hazards that too often harm rather than help patients.

In response to this evolving discussion, the International Society for Quality in Healthcare recently nominated an Innovations Team that is now developing new strategies. One of the emerging themes is that the medical field cannot resolve this problem on its own. Instead, interdisciplinary collaborations are needed to advance effective, evidence-based interventions that will eventually result in competent changes.

In March 2013, the Institute of Communication and Health at the University of Lugano organized a conference on Communicating Medical Error (COME 2013) in Switzerland to stimulate such interdisciplinary dialogue. International scholars from eight disciplines and 17 countries attended the congress to discuss interdisciplinary ideas and perspectives for advancing safer care. The team of invited COME experts collaborated in compiling this issue of the Journal of Public Health Research entitled Interdisciplinary perspectives on medical error. This particular issue introduces relevant North American and European theorizing and research on preventable adverse events. The caliber of scientists who have contributed to this issue is humbling. But rather than naming their affiliations and summarizing their individual manuscripts here, it is more important to reflect on the contribution of this special issue as a whole. Particularly, I would like to raise two important take-home messages that the articles yield: i) What new insights can be derived from the papers collected in this issue? ii) What are the central challenges implied for future research on medical error?

\section{Medical error: a highly complex phenomenon that requires interdisciplinary collaboration}

The overarching theme that emerges from this special issue is that patient safety is complex and will remain so. In fact, the degree of its complexity is so extensive that it cannot possibly be captured by any single one of the eight disciplinary perspectives that are represented in this issue (i.e., medicine, communication science, human factors science, law, psy- chology, health policy, information technology, and the insurer perspective). This complexity manifests itself in systemic, interpersonal, and personal layers that are embedded within technological, cultural and legal contexts. This special issue, then, is a first attempt to capture and communicate the complexity of this multilayered phenomenon by approaching it from a rich interdisciplinary diversity. Thus: John Petrocelli and Brian Spitzberg illuminate personal factors in their respective contributions; Sandra Petronio as well as Albert Wu, Dennis Boyle, Gordon Wallace, and Kathleen Mazor raise the interpersonal complexity of medical errors; Jane Carthey and Tanja Manser present systemic perspectives on human error in medicine; and last but not least, Julius Pham, Thierry Girard, Peter Pronovost, John Clarke, Harold Thimbleby and Olivier Guillod cover the cultural, technological and legal issues that define the contextual atmosphere within which medical errors take place. All these perspectives illuminate the complexity of the field. The take-home message is that medical error cannot be reduced exclusively to systemic, interpersonal, or personal factors alone. Instead, each of these perspectives are only single components of the whole picture, and an isolated intradisciplinary perspective would not do full justice to the true nature of the problem.

\section{Central challenges in the scientific pursuit of evidence-based medical error research}

There are five overarching central challenges that can be derived from the articles presented in this special issue. Each of these challenges entails a dialectical tension, which opens new grounds for heuristic thinking that may help direct future investigations on medical error into different paradigms.

\section{Goal-setting: human nature versus error-free care}

The Hippocratic dictum that guides medical practice obligates physicians to first, do no harm. However, to err is human, and thus human errors in medicine have always been and will always remain an unavoidable issue. Once we embrace this, we will recognize that automation cannot be a reasonable end-goal for patient safety agendas. Furthermore, we will recognize that we will never achieve complete satisfaction with respect to safety of care. The question that arises is where we should set the line that distinguishes acceptable satisfaction from unacceptable dissatisfaction. In other words, when does the glass become half empty rather than half full? This line is difficult to define and deserves some discussion. The takehome message is that there is no perfect system within which humans will no longer make mistakes. Humans will continue to err, and no personal, interpersonal, or systemic intervention will prevent that. Thus, research will never come to an end in pursuing patient safety advancements.

\section{Translation: knowledge advancement versus best-practice implementation}

Evidence-based medical practice, technological advancements as well as legal regulatory changes accumulate quickly. At the same time, this rapid change is not always visible to physicians, and they may not have adequate 
access to training that would allow them to quickly and effectively adapt these new developments into practice. Thus, while expectations and standards of care advance quickly, physicians are not reacting to them at the same speed and increasingly lag behind. In this light, it is no surprise that checklists are not practiced in every hospital in the world, or that only the minority of physicians will know to what extent their error disclosure is legally protected in their respective states and countries. Given the daily pressures of their job, how could they possibly follow and then find ways to change their practice patterns? And even if they could, can we guarantee that their adoption of new routines might not cause more harm to patients given that clinicians suddenly have to perform outside their familiar practice patterns? For example, may IT-advancements introduce new, if not higher probabilities of erring, given a physician's initial lack of skill with new equipment? And may perceptions of uncertainty regarding recent legal changes restrict rather than enlighten medical practice, so that physicians practice defensive rather than safe (i.e., legally protected) medicine? The bottom line is that there is a considerable time lag between reliable knowledge accumulation and effective behavioral change, and it is important to reflect on whether the improvements physicians need may actually increase error rather than reduce it.

\section{Ethics: dignity versus failure}

Immanuel Kant defended human dignity by arguing that we need to treat persons as ends in themselves rather than means to some other end. ${ }^{2}$ In the context of a medical error, this respect for autonomy and dignity is complex. To this date, research has predominantly focused on the consequences of medical errors on patients. However, medical errors also generate a need for the provider to reestablish dignity. How this need is fulfilled primarily depends on the patient. Thus, unlike in any other setting, the context of medical error creates an existential interdependency that has not been properly discussed in the literature so far. It extends recent second victim discussions (the second victim emphasizes that both provider and patient have fallen victim to human error). ${ }^{3}$ But at this point, a key question emerges: how can the dignity of the physician who has erred be effectively restored? Thus, in the context of medical error, dignity is no longer a right, but it is a relational need. The ethical practice of dignity therefore becomes intrinsically complex in this interdependent setting, particularly if the error is attributed to a particular person rather than to human nature (or to the wider system in which the person operates).

\section{Contextualization: human needs versus cultural needs}

There are human needs that are generalizable across cultures. Maslow categorized a well-known hierarchy that includes: physiological (e.g., food, sleep, shelter), safety (e.g., resources, income, job security), social (e.g., relationships, intimacy, belonging), esteem (e.g., achievement, respect, status), and self-actualization (e.g., personal growth, authenticity) needs. ${ }^{4}$ However, such needs are embedded within cultural contexts, and cultural contexts may modify or suppress behaviors that would express such fundamental needs. It is important, then, to acknowledge that whereas the human needs that are threatened by a medical error are similar across cultures, the cultural frameworks that surround a physician's practices will influence physician's behaviors. This tension becomes particularly evident when US-centered patient safety research is implemented in other cultures without consideration that the results and promised effects may not simply replicate in a different cultural environment. The take-home message of this fourth tension is that human needs do not always align with cultural needs, and this challenge needs to be considered in scientific investigations that aim to improve the quality of medical care.

\section{Patient-centeredness: evidence versus conscience}

The theme of patient-centeredness is a predominant focus in patient safety efforts. In fact, the International Society for Quality in Healthcare recently selected this term as a focus to improve quality care. However, a concep- tual discussion of what constitutes patient-centered care needs to occur. Is medical practice patient-centered when it performs according to best knowledge (i.e., evidence-based guidelines), or when it performs according to best conscience (i.e., the patient's wishes and needs)? At times, these two elements stand in contradiction, so it is important to define whether the medical perspective or the patient perspective should be used as the measure to assess patient-centered care.

Communication is the vehicle through which a leveraging of these tensions can take place. A primary goal of communication is to establish shared meaning. Thus, if shared mutual meaning has been achieved, communication has been effective, even if it consists of disagreement. It is through communication that individuals define their relationships with others, and their communication indicates the degree of participation they are willing to engage in to establish such shared meanings. Thus, communication is a fundamental practice in achieving patient-centered care, and it - if performed competently - is the bridge that will unite the dimensions to become complementary.

\section{Concluding thoughts}

Research in medicine often focuses on human performance and systemic prerequisites for safer care. But do we gain a better understanding of the broader nature of human error? What should be the determining, predefining measures of medical error - should we orient ourselves by the level of harm? Or should we focus on deviant behavior, and if so, which measure should we use to judge deviance? Or should we focus on human nature to better understand medical errors? Cultural, legal, technological, systemic, interpersonal, and personal perspectives alone cannot deliver answers to any of these questions. In order for our fundamental understanding of error to advance, we must recognize the complexity of the problem and identify common grounds on which we can build fruitful interdisciplinary collaborations to enhance medical care. And this is the basic purpose of this special issue. I would like to thank the influential scholars and practitioners who have put their minds to this challenge. I hope that this special issue will only be the beginning of this interdisciplinary exchange, and that it will have a contagious effect on continuous and expanding collaborations that aim to make health care safer and less threatening for all parties involved.

Correspondence: Prof. Annegret F. Hannawa, Faculty of Communication Sciences, Institute of Communication and Health, University of Lugano, via G. Buffi 13, CH6904 Lugano, Switzerland. Tel. +41.058.666.4482 - Fax: +41.058.666.4647.

E-mail: annegret.hannawa@usi.ch

Received for publication: 1 November 2013.

Accepted for publication: 1 November 2013.

CC Copyright A.F. Hannawa, 2013

Licensee PAGEPress, Italy

Journal of Public Health Research 2013; 2:e22

doi:10.4081/jphr.2013.e22

This work is licensed under a Creative Commons Attribution NonCommercial 3.0 License (CC BY-NC 3.0).

\section{References}

1. Jha AK, Larizgoitia I, Audera-Lopez C, et al. The global burden of unsafe medical care: analytic modelling of observational studies. BMJ Qual Saf 2013;10:809-15.

2. Kant I. Grundlegung zur Metaphysik der Sitten. 1870.

3. Wu AW. Medical error: the second victim. The doctor who makes the mistake needs help too. BMJ 2000;320:726-7.

4. Maslow AH. A Theory of human motivation. Psych Rev 1943;50:370-96. 